INPLASY

PROTOCOL

To cite: Hou et al. Acupuncture for anosmia in COVID-19,A protocol for systematic review and meta-analysis. Inplasy protocol 2021120134. doi: 10.37766/inplasy2021.12.0134

Received: 30 December 2021

Published: 30 December 2021

Corresponding author: Yi Hou

hy1994315@163.com

Author Affiliation:

Shandong University of TCM.

Support: Shandong University of TCM.

Review Stage at time of this submission: Preliminary searches.

Conflicts of interest:

None declared.

\section{Acupuncture for anosmia in COVID-19, A protocol for systematic review and meta-analysis}

Hou, Y1; Li, X²; Dong, T3; Wang, X4; Yang, J5.

Review question / Objective: How do acupuncture treat COVID-19 anosmia?

Condition being studied: A systematic evaluation of the effect of acupuncture on COVID-19 anosmia.

Information sources: Randomized controlled trials will be extracted from PubMed, EMBASE, Cochrane Library, Web of Science, Chinese Biomedical Databases, China National Knowledge Infrastructure, Wanfang Database, and VIP Database.

INPLASY registration number: This protocol was registered with the International Platform of Registered Systematic Review and Meta-Analysis Protocols (INPLASY) on 30 December 2021 and was last updated on 30 December 2021 (registration number INPLASY2021120134).

\section{INTRODUCTION}

Review question / Objective: How do acupuncture treat COVID-19 anosmia?

Condition being studied: A systematic evaluation of the effect of acupuncture on COVID-19 anosmia.

\section{METHODS}

Participant or population: Patients with COVID-19 anosmia.
Intervention: The intervention measures in the experimental group should contain acupuncture.

Comparator: As included in eligible randomized clinical trials and retrospective cohort studies.

Study designs to be included: Randomized clinical trials and retrospective cohort studies will be included. 
Eligibility criteria: Subjects with documented COVID-19 with anosmia lasting four weeks or longer There were no restrictions on sex, race, or disease stage. Patients with a history of anosmia prior to the COVID19 infection were excluded.

Information sources: Randomized controlled trials will be extracted from PubMed, EMBASE, Cochrane Library, Web of Science, Chinese Biomedical Databases, China National Knowledge Infrastructure, Wanfang Database, and VIP Database.

Main outcome(s): $\mathbf{T}$ \& $\mathbf{T}$ olfactometer test score; Sniffin'sticks olfactory stick test score;University of Pennsylvania olfactory recognition test score; The quality of life.

Quality assessment / Risk of bias analysis: The methodological quality of each included study will be assessed independently by two reviewers using two tools. The Cochrane collaboration tool will be used to assess the quality of randomized controlled trials. It comprises the following 7 aspects: random sequence generation, allocation concealment, blind method, incomplete result data, selective reporting, and other biases. The quality assessment results of each item can be divided into three grades: "low risk", "high risk" and "unclear". The more rigorous the design and the higher the methodological quality of each RCT, the lower the risk coefficient.The Newcastle Ottawa Scale (NOS) will be used to assess the quality of retrospective studies. This method includes three aspects to the evaluation: the selection method, comparability and contact exposure assessment method of case group and control group. The higher the score, the higher the quality of the study. When necessary, the consensus on this issue will be studied with the help of a third party.

Strategy of data synthesis: The RevMan5.3 software provided by the Cochrane website will be used for the analysis. Categorical variables will be expressed by odds ratio (OR) and marked with $95 \%$ confidence interval $(\mathrm{Cl})$. Continuous variables will be expressed by mean difference (MD) and marked with $95 \% \mathrm{Cl}$. If $\mathrm{PO} .1$ or $\mathrm{I}^{2}<50 \%$, it means that the heterogeneity between groups is small, and the fixed effect model will, in this case, be used for combined analysis; when $\mathrm{P} 50 \%$, it shows that the heterogeneity between the groups is large, and the random effects model will then be used for combined analysis, and the results shown in forest plots.Analysis of potential publication bias will be shown in a funnel chart, and sensitivity analysis and subgroup analysis will be used when necessary.

Subgroup analysis: Subgroup analysis will be used to evaluate the therapeutic effects among different drugs.Inverted funnel plots and Egger's regression test will be used to determine publication bias when the number of included studies exceeds 10 in the network meta-analysis.

Sensitivity analysis: Sensitivity analyses will be performed to determine whether the results are affected by leave1out with Stata14.0.

Country(ies) involved: China.

Keywords: acupuncture, meta-analysis, protocol, COVID-19, anosmia.

Contributions of each author:

Author 1 - Yi Hou.

Author 2 - Xuhao Li.

Author 3 - Tiantian Dong.

Author 4 - Xingxin Wang.

Author 5 - Jiguo Yang. 